\title{
An Entropy-Based Analysis of GPR Data for the Assessment of Railway Ballast Conditions
}

\author{
F. BENEDETTO ${ }^{1 *}$, IEEE, Senior Member, F. TOSTI ${ }^{2}$, M. (Amir) ALANI ${ }^{2}$
}

Abstract - The effective monitoring of ballasted railway track beds is fundamental for maintaining safe operational conditions of railways and lowering maintenance costs. Railway ballast can be damaged over time by the breakdown of aggregates or by the upward migration of fine clay particles from the foundation, along with capillary water. This may cause critical track settlements. To that effect, early stage detection of fouling is of paramount importance . Within this context, ground penetrating radar (GPR) is a rapid, non-destructive testing (NDT) technique, which is being increasingly used for the assessment and the health monitoring of railway track substructures. In this study, we propose a novel and efficient signal processing approach based on entropy analysis, which was applied to GPR data for the assessment of the railway ballast conditions and the detection of fouling. In order to recreate a real-life scenario within the context of railway structures, four different ballast/pollutant mixes were introduced, ranging from clean to highly-fouled ballast. GPR systems equipped with two different antennas, ground-coupled (600 and $1600 \mathrm{MHz})$ and air-coupled (1000 and $2000 \mathrm{MHz}$ ), were used for testing purposes. The proposed methodology aims at rapidly identifying distinctive areas of interest related to fouling, thereby lowering significantly the amount of data to be processed and the time required for specialist data processing. Prominent information on the use of suitable frequencies of investigation from the investigated set, as well as the relevant probability values of detection and false alarm, are provided.

Index Terms - Ground penetrating radar; entropy; railway ballast; ballast fouling, performance analysis.

\footnotetext{
${ }^{* 1}$ Signal processing for Telecommunications and Economics Lab., Roma Tre University, Via Vito Volterra 62, 00146, Rome, Italy; e-mail: francesco.benedetto@uniroma3. francesco.benedetto@uniroma3.it)
}

${ }^{2}$ School of Computing and Engineering, University of West London (UWL), St Mary's Road, Ealing, London W5 5RF, UK 


\section{INTRODUCTION}

Ballasted railroads are among the most common construction types in railway engineering due to the effective drainage capability and the load-bearing capacity achieved at relatively low construction costs. Overall, a track structure can be divided into the superstructure (steel rails, fastening systems and concrete/timber sleepers) and the substructure (ballast, subballast and subgrade). The interaction between these two main components affects the safety conditions and the quality of ballasted rail tracks, as both are influenced by the cyclic loading exerted by moving trains [1]. Thereby maintenance and rehabilitation costs may be heavily affected by the ineffective interaction between these two components.

The primary task of ballasted track beds in railways is to distribute the load from the sleepers to the capping layers and formation soils at a reduced and suitable stress level [2]. Rail ballast is usually made of uniformly-graded coarse aggregates derived from crushed rocks of differing geological nature, mostly granite, basalt and limestone. Amplitude and number of load cycles, track confining stress, grain size of the aggregates and the angularity and fracture strength of individual grains are the main factors which may cause ballast fouling and differential track settlement. According to Selig and Waters [2], several classes can be identified as principal source mechanisms of fouling: i) the breakdown of ballast, ii) the infiltration from the ballast surface (downward migration of coal dust from commercial trains) and iii) the upward migration of clay fines from the subgrade, are the major causes of fouling. Notwithstanding the increased costs of maintenance, fouling occurrence may dramatically impact on the safety and the operation of railways [3]. In view of this, effective health monitoring and early-stage detection of fouling is mandatory to allow significant reduction of both unsafe events and maintenance costs [4]-[6]. Numerous studies dealt with the assessment of fouling and several methods have been identified [2], [7]-[8]. The major drawback of these techniques lies in the need of digging trenches and testing the material in the laboratory environment. These operations are costly and inefficient. Within this context, non-destructive testing (NDT) techniques are becoming more important in the health monitoring of railways. In particular, fouling inspection of ballasted railroads have been carried out using infrared imaging [9], electrical resistivity tomography [10], seismic surveys [11] and, mostly, ground penetrating radar (GPR) [12]-[14].

The first GPR applications in railway engineering date back to the nineteen eighties [15] and involved low-frequency systems mounted over the interaxis of the rails [16]. The GPR sends an electromagnetic (EM) wave into the ground using a transmitting antenna and receiving the back-reflected signal by a 
receiver antenna in a given frequency band [17]. Overall, the interfaces between different materials within the railway track bed and the inhomogeneities within the materials with different EM properties cause changes in the signal peak positions and amplitudes. These changes are converted into information on the layer thicknesses and properties of the materials [13], [18]-[19]. In general, a set of antenna frequencies is used under controlled boundary conditions for investigating same track bed scenarios. Two types of approach can be distinguished, namely, $i$ ) methods based on the analysis of the signal response in the time domain, and ii) methods based on the spectral analysis of the signal in the frequency domain. The first class includes mostly analyses of single or multiple (i.e., radar scans) traces for the thickness evaluation of the rail substructure and the detection of main anomalies (e.g., fouling). The evaluation of the permittivity of the medium passed through [20]-[21] and the analysis of the signal amplitudes versus scattering from the voids [22]-[23] are usually performed. The results are compared with real-truth data extracted from laboratory tests, full-scale track beds at the test-site scale, or local trenches in the real-life environment. The frequency-based research has been focused on the spectral analysis of the GPR signals. Leng and Al-Qadi [24] used the Short-Time Fourier Transform (STSF) to track the frequency spectrum change with time, and graphically demonstrated the frequency energy variation with the depth of the ballast under differing conditions. This STSF method indirectly provides information about the ballast fouling conditions. Shao et al. [25] presented an automatic classification system to assess the railway ballast conditions, based on the extraction of magnitude spectra at salient frequencies and their classification, using support vector machines. The system was capable of distinguishing GPR signals reflected by the different ballast conditions. Xiao and Liu [26] applied the forward and inverse S-transform to a dual-frequency data set made of 100and 400-MHz GPR data, and achieved a spectrum with broader bandwidth after fusing together the GPR spectra from both the frequencies. Subsequently, the synthesized spectrum was converted back to a time-domain signal and proved an effective trade-off between good resolution and deep penetration in ballast inspections. The use of finite-difference time-domain (FDTD) simulation of the GPR signal was recently demonstrated to be effective in the interpretation of complex scenarios, and the validation of the results from the aforementioned processing methodologies [27]-[28]. Although the above approaches have proved high effective and reliable results, they require high computational times and higher costs. This occurs especially in railway investigations, as they are characterized by huge amounts of data collected within the infrastructure development.

This paper reports on a signal processing approach, based on the entropy analysis of the radar signal, aimed at investigating the railway ballast conditions and detecting the presence of fouling. To this 
effect, a laboratory set-up was built and both cleaned and fouled ballast scenarios were manufactured. GPR tests were carried out using ground-coupled and air-coupled GPR systems equipped with 600-, 1000-, 1600-, and 2000-MHz frequency antennas. The proposed methodology aims at rapidly identifying distinctive areas of interest related to fouling, thereby lowering significantly the amount of data to be processed and the time required for further data processing.

The remainder of the paper is organized as follows. In Section 2, the GPR working principles as well as the system model are discussed. Section 3 illustrates the entropy-based procedure for the assessment and the monitoring of the railroad ballast conditions. In particular, the first half of the section includes the rationale of our work, whereas the second half provides the mathematical details of the proposed entropy-based test. The initial part of Section 4 describes the laboratory set-up for the GPR measurements; the numerical results are instead outlined in the second half of the Section. Finally, the conclusions and future perspectives are discussed in Section 5.

\section{THEORETICAL FRAMEWORK}

\subsection{GPR working principles}

A GPR system is usually composed by one or more transmitter and receiver antenna(s), a radar control unit, and suitable data storage and display devices. The transmitter antennas emit an electromagnetic impulse which is then collected by the receiver antennas, after being reflected and scattered by any dielectric anomaly in the subsurface. The time delays, the frequency modulations and the amplitudes of the reflected signals are effective indicators to evaluate the characteristics of the materials. The collected information is then down-converted to a low-frequency signal, such that they can be digitized by a conventional analog-to-digital (A/D) converter for further processing and display. Measurements can be traditionally performed in ground-coupled or air-coupled antenna configurations, as a function of the main purpose and type of the survey. The antennas are chosen according to the required depth of penetration, soil type and size of the anomalies to detect. During a railway ballast inspection, a GPR scan is usually performed as a sequence of vertical radar profiles (or sweeps). Subsequently, these 1-D measurements are used to create a 2-D matrix, i.e., the datagram; this is used for the visual interpretation of the acquired data directly from the acquisition device. The datagram is represented with the depth step (usually measured in centimeters) on the vertical axis, and the longitudinal step (usually measured in meters) on the horizontal one. A return at a certain position 
along the distance axis is named A-scan, which is represented with the depth (time) step on the vertical axis and the amplitude of the signal (measured in Volts) on the horizontal one (Figure 1).

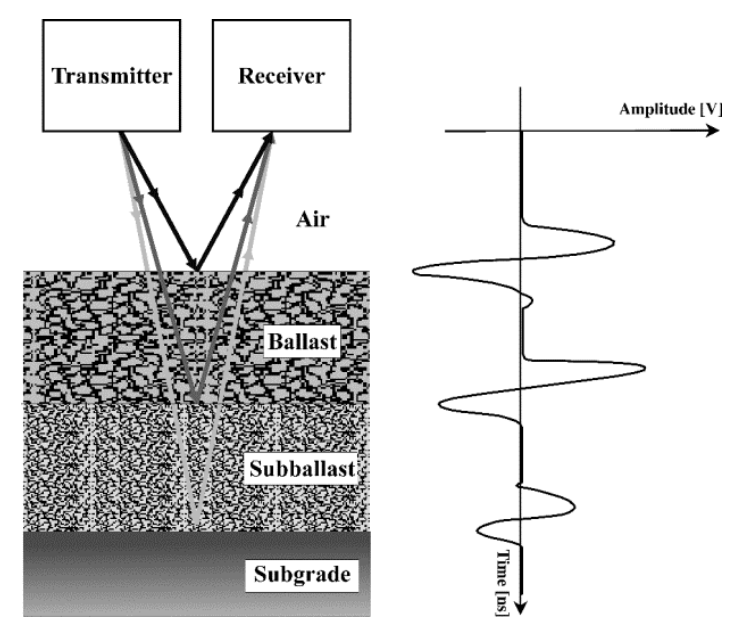

Fig. 1. Cross-section of a railway substructure and the typical reflection pattern from a GPR measurement (A-scan).

\subsection{GPR analytical model}

The received signal $x(t)$ can be analytically modelled in the continuous time domain as the sum of several scaled and delayed replicas of the GPR transmitted electromagnetic pulse plus the noise. The considered model follows the general equation [29]:

$x(t)=\sum_{i=1}^{M} h_{i} \cdot s\left(t-\tau_{i}\right)+w(t)$

where $s(t)$ is the transmitted pulse, $x(t)$ is the received pulse composed of $M$ replicas of $s(t)$ with different amplitudes $\left\{h_{i}\right\}_{i=1, \ldots, M}$ and delays $\left\{\tau_{i}\right\}_{i=1, \ldots, M}$, and $w(t)$ is the random noise (i.e., the noise due to the scattering from the buried objects and the clutter phenomena). The signal $x(t)$, obtained as the sum of a random noise process (the noise due to the fouled ballast) and a deterministic signal (i.e. the original GPR pulse), can be modelled as a stochastic (or pure random) process [30]. In fact, for practical scenarios it can be overly simplistic to exploit only deterministic modelling of radar signals [31]. There can be fading or dispersion in signal propagation and, in some cases, the signal sources can be correlated, either by nature or as a result of some modelling practice [32]. In those cases, it is more appropriate to treat target sources as random and characterize them by their statistical properties [33]. In the case of our interest, the autocovariance function is exploited to study and analyse the received GPR signal [34]. The autocovariance function allows to focus on the nature of the noise which is the source of randomness in this process, by applying the maximum entropy method as described in the next Sections. At the receiver side, the signal $x(t)$ in (1) is then sampled (with a 
sampling rate $T_{s}$ ) and the sequence $x(n)$, with $n=1, \ldots, N$, is obtained for further processing in both the time and the frequency domains. In particular, let us now define with $\operatorname{Cov}(k)$ the autocovariance function of the series $x(n)$ of $N$ data, and defined as:

$\operatorname{Cov}(k)=\frac{1}{N} \sum_{n=1}^{N} x(n) \cdot x^{*}(n-k)-|\mu|^{2}$

where $k=-N, \ldots,+N, x^{*}(n)$ stands for complex conjugate, and the mean $\mu$ is expressed by:

$\mu=\frac{1}{N} \sum_{n=1}^{N} x(n)$

The autocovariance function can be analysed in the transformed (frequency) domain by the application of a discrete Fourier transform, thereby obtaining the power spectral density PSD, $S\left(\omega_{k}\right)$, given by Oppenheim and Schafer [34]:

$S\left(\omega_{n}\right)=\sum_{k=-N}^{N} \operatorname{Cov}(k) \cdot e^{-j \cdot \omega_{n} \cdot k}$

where $\omega_{n}=\frac{2 \cdot \pi \cdot n}{N}$.

A growing number of GPR-related studies are nowadays oriented towards analyses performed in the spectral domain of the signal. To that effect, reliable and robust indexes are usually retrieved from the frequency spectrum of the signal for the correlation with critical physical parameters, such as water [35], clay [36]-[38], or coal-based fouling [25], which may have major impacts on the load-bearing capacity of the subgrade soils in transportation infrastructures.

\section{Entropy-BASEd Testing Method}

\subsection{Analytical model}

The idea behind our work is to investigate the presence of fouling in railway ballast by means of an entropy-based analysis. Entropy is a concept initially borrowed from the classical mechanics, and later from the information theory. In mechanics, the entropy is used to quantify the disorder and the uncertainty of dynamical systems, or in other words, it is an expression of the randomness of a system [39]. On the other hand, the entropy in information theory is considered as a measure of the information content of the series under investigation [40]. In particular, Shannon [40] has also related the concepts of entropy and uncertainty in his pivotal work. He finally argued that the information is a measure of the degree of uncertainty exercised by the source in the phase of selecting the message to transmit (i.e. the presence of a regular pattern in the ballast/assessing good as-built conditions, herein). In the definition of Shannon, given a source $X$ that can transmit $N$ symbols (where each $i$-th symbol is characterized by an a-priori probability $\left.p_{i}\right)$, the entropy $H(X)$ is defined as: 
$H(X)=-\sum_{i=0}^{N-1} p_{i} \cdot \log _{2} p_{i}$. The Shannon entropy equation provides a way to estimate the average minimum number of bits needed to encode a string of symbols (emitted by a source $X$ ), based on the frequencies of the symbols. If one of the symbols is more probable than others, the observation (i.e. reception) of that symbol is less informative for the receiver. Conversely, rarer symbols provide more information when observed. Since observation of less probable symbols occurs more rarely, the net effect is that the entropy (thought of as average information) received from non-uniformly distributed data is less than $\log _{2}(N)$. The entropy is zero when one outcome is certain. In practical terms, the information is the removal of the uncertainty: high values of the Shannon entropy are relevant of an increasingly polluted ballast (by fouling intrusion), whereas lower values mean less uncertainty; hence, a more regular and unpolluted ballast. The entropy was used in several fields of application such as the biomedical engineering, speech, information data mining, front-wall clutter rejection, financial signal processing, and color image enhancement. It is well known that a GPR radargram is usually two-dimensional (2-D). Moreover, in many applications several 2-D radargrams are combined together to provide a three-dimensional (3-D) representation. To the best of our knowledge, there is only one paper which applies the entropy analysis to GPR systems by the use of the 2-D entropy of GPR images for detection of sporadically distributed features [41]. Conversely, we are interested in providing a fast and low-complex entropy-based algorithm to quickly assess the railway ballast conditions; hence, we are focusing on a simpler one dimensional (1-D) system model, as described in eqs. (1)-(4).

The rationale of our approach is as follows: if the ballast is a "regular one" (i.e. without intrusions of fines), its structure should be characterized by repetitive patterns, hence its entropy should tend to small values. Conversely, if the ballast is fouled (i.e. it is a "polluted" ballast), its structure becomes irregular, and the entropy of the system should reach higher values. In the proposed approach, the term "repetitive" stands for periodic structure, such as the one representing the clean ballast conditions. On the other hand, the term "irregular" means non-periodic, such as the structure representing fouled ballast [42]. Thereby, if the ballast is clean, its structure is repetitive (the same pattern repeated in the structure), and it can be represented (or "encoded", speaking in terms of information) by a few bits (low values of entropy). On the contrary, if the ballast is polluted, its structure contains more information, hence it should be encoded with a higher number of bits (i.e. a higher entropy value than in the "clean ballast" case). 


\subsection{Entropy of a GPR radargram}

Conventional entropy theories are usually related to infinite data series, which correspond to an infinitely accurate precision and resolution for the evaluation of the entropy. Nevertheless, practical data are finite time series data, sampled with a sampling rate $T_{s}$ and characterized by a limited resolution. The main issue is that an accurate estimation of the entropy series requires a considerable amount of data to be processed, and the results will be greatly influenced by the system noise. In 1967, Burg [43] proposed a new approach within the field of the spectral estimation. The author attempted to derive a procedure for achieving a high resolution where only a few data of the estimates of an autocorrelation sequence were available [44]. This has been defined as the maximum entropy method (MEM). The MEM method is capable to provide the highest frequency resolution, compared with the auto-correlation and covariance methods [45], by relating the entropy rate of a time series with its power spectral density (PSD). Hence, by knowing the PSD of a series, it allows to know its entropy rate. Thereby, if we denote with $S(\omega)$ the PSD of the received GPR time series, given by (4), the entropy rate $h$ (in the following referred to as entropy) is given by [46]:

$h=\frac{1}{2} \ln (2 \cdot \pi \cdot e)+\frac{1}{4 \cdot \pi} \cdot \int_{-\pi}^{\pi} \ln (2 \cdot \pi \cdot S(\omega)) \cdot d \omega$

where $\ln (\cdot)$ is the natural logarithm (i.e. with base $e$ ) and $e$ is the Euler's number. It is important to emphasize that the entropy of a finite segment of a stochastic process is upper-bounded by the entropy of a segment of a Gaussian random process, according to (5). This means that a white time series (i.e. a received GPR signal representing only uncorrelated noise) is characterized by the maximum entropy. Therefore, lower entropy values return more repetitive (and correlated) series. Thereby, the entropy can be used as an indicator of the presence of repetitive patterns in a ballasted track bed and, hence, for the assessment of the ballast conditions in terms of performance.

\subsection{Entropy test for the assessment of the railway ballast conditions}

The theoretical approach of the entropy-based test for the assessment of the railway ballast conditions is based on the classical Neyman-Pearson radar test implemented with a constant false alarm rate (CFAR) strategy [47]. Indeed, the test here proposed is a binary decision problem where each of the two outputs corresponds to one hypothesis. The proposed test distinguishes between two different hypotheses: the polluted ballast condition (hypothesis $H_{l}$ ), which corresponds to the case of intrusion of fine-graded material within the ballast voids (i.e. fouled ballast condition); and the clean ballast case (hypothesis $H_{0}$ ), which conversely represents the absence of fine-graded material intrusion in the analysed scenario. Hence, the decision about the presence or absence of material intrusion within the ballast voids is obtained by comparing a decision metric (or testing variable) against a fixed threshold. 
The testing variable of our test is represented by the entropy of the GPR railway scan, evaluated according to (5). In particular, since the GPR sweeps in a railway survey can be affected by many sources of noise and their signal-to-noise ratio (SNR) values can significantly decrease, we evaluate the testing variable as the average entropy of all the radar sweeps of that particular GPR scan. In other words, the GPR traces are combined together using the spatial dimension for averaging, while exploiting the temporal axis for the entropy evaluation. From a mathematical point of view, if a GPR datagram is composed (along the spatial dimension) by $Q$ traces of $N$ samples each, the estimated average entropy is given by:

$H=\frac{1}{Q} \sum_{q=1}^{Q} \widehat{h_{q}}$

where $\widehat{h_{q}}$ is the estimated entropy of the $q$-th radar sweep, evaluated as follows (along the temporal dimension):

$\widehat{h_{q}}=\frac{1}{2} \ln (2 \cdot \pi \cdot e)+\frac{1}{4 \cdot \pi} \cdot \sum_{n=-N}^{N} \ln \left(2 \cdot \pi \cdot S\left(\omega_{n}\right)\right)$

Therefore, considering a threshold $\eta$, the test is expressed as follows:

Null Hypothesis: $\quad H<\eta$,

Alternate Hypothesis: $\quad H \geq \eta$

The above set relationship means that if the testing variable is greater than the threshold $\eta$, the algorithm decides for $H_{l}$ (i.e. presence of fouling); else, the choice is for $H_{0}$ (i.e. absence of fouling).

\subsection{Performance analysis}

Considering a set of $Q$ observations $R\left(r_{1}, r_{2}, \ldots, r_{Q}\right)$ and considering the decision domains $Z_{0}$ (absence of fouling) and $Z_{1}$ (presence of fouling), it is possible to evaluate the probability of detection $\left(P_{D}\right)$ and the probability of false alarm $P_{F}$, as follows:

$P_{D}=\int_{Z_{1}} p_{r \mid H_{1}}\left(R \mid H_{1}\right)$

$P_{F}=\int_{Z_{1}} p_{r \mid H_{0}}\left(R \mid H_{0}\right)$

where $p_{r \mid H_{i}}\left(R \mid H_{i}\right)$, with $i=1,2$, is the probability density function of the observations conditioned to, respectively, the null and the alternate hypothesis. The subscripts were chosen in accordance with the classical radar problem, where $H_{1}$ corresponds to the presence of a target (i.e. the presence of fouling) and $H_{0}$ to its absence. $P_{F}$ is the probability of a false alarm (i.e. we find that pollution is 
present when it is not) and $P_{D}$ is the probability of detection (i.e. we find that pollution is present when it is). In this case, (9) and (10) can be rearranged as follows:

$$
\begin{aligned}
& P_{D}=p\left(\widehat{H_{1}} \mid H_{1}\right) \\
& P_{F}=p\left(\widehat{H_{1}} \mid H_{0}\right)
\end{aligned}
$$

where the symbol $p\left(\widehat{H_{l}} \mid H_{j}\right)$ with $i=j$ or $i \neq j$ is the probability of the hypothesis $\widehat{H_{l}}$, numerically computed from the observation, assuming that the hypothesis $H_{j}$ is true. The above probabilities thoroughly describe the performance of such Neyman-Pearson tests. As it is well known, the two objectives of reducing $P_{F}$ while increasing $P_{D}$ play a contrasting role. The used strategy constrains one of the probabilities while it maximizes (or minimizes) the other. In traditional GPR applications, $P_{F}$ is constrained to be $P_{F}=\alpha^{\prime}<\alpha$ where $\alpha$ is the accepted reliability standard while the objective is to maximize $P_{D}$, which conversely determines the system performance. It is worth to emphasize that the testing variable in $(6)$ is asymptotically $(Q \rightarrow \infty)$ Gaussian according to the central limit theorem. The threshold $\eta$ can be asymptotically tuned from a straightforward evaluation of the Gaussian integral for a fixed probability of false alarm. Under this condition, the mean $E\left[H \mid H_{0}\right]$ and the variance $\operatorname{var}\left[H \mid H_{0}\right]$ are evaluated under the null hypothesis to compute the threshold:

$$
\eta=E\left[H \mid H_{0}\right]+\left(2 \cdot \operatorname{var}\left[H \mid H_{0}\right]\right)^{1 / 2} \cdot \operatorname{erf}^{-1}\left(1-2 \cdot P_{F}\right)
$$

whereas the mean $E\left[H \mid H_{1}\right]$ and the variance $\operatorname{var}\left[H \mid H_{l}\right]$ conversely determine the asymptotic $P_{D}$ :

$P_{D}=\frac{1}{2}+\frac{1}{2} \cdot \operatorname{erf}^{-1}\left[\left(-\eta+E\left[H \mid H_{1}\right]\right) \cdot\left(2 \cdot \operatorname{var}\left[H \mid H_{1}\right]\right)^{-1 / 2}\right]$

where $\operatorname{erf}^{1}(\cdot)$ is the inverse of the well-known Gaussian error function.

\section{Results}

\subsection{Performance analysis}

Investigations on railway ballast material were carried out within a square-based methacrylate tank with $155 \mathrm{~cm}$ long base sides and height of $55 \mathrm{~cm}$ (outer dimensions) and relevant inner dimensions of $148 \mathrm{~cm}$ and $48 \mathrm{~cm}$, respectively. A $2 \mathrm{~m} \times 2 \mathrm{~m}$ copper sheet, acting as a perfect electric conductor (PEC), was placed underneath the tank to allow the complete reflection of the waves propagating through the material as well as to avoid any unwanted reflection from the ground of the laboratory (Figure 2). The measurements were performed using ground-coupled and air-coupled pulsed GPR systems manufactured by IDS Georadar. 


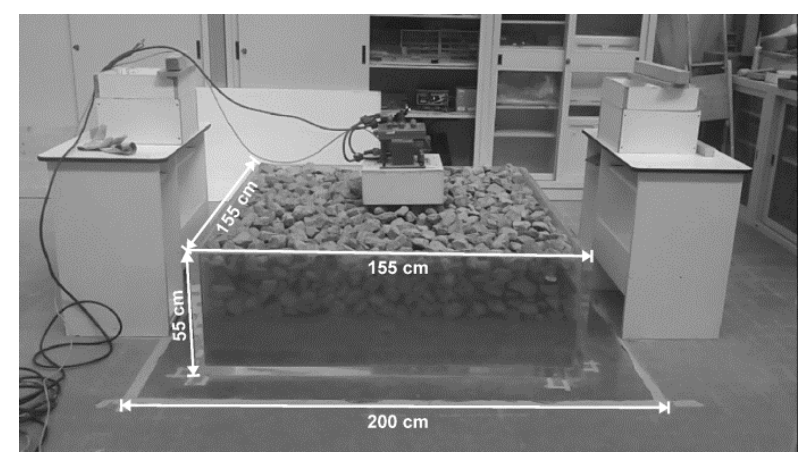

Fig. 2. Experimental setup for the laboratory measurements.

The ground-coupled multi-frequency GPR system RIS 99-MF, equipped with $600 \mathrm{MHz}$ and 1600 $\mathrm{MHz}$ monostatic antenna frequencies, was set to collect 512 sample size data with a time window of $40 \mathrm{~ns}$ (time step of acquisition equal to $7.83 \times 10^{-2} \mathrm{~ns}$ ). In addition, three air-coupled GPR systems, equipped with 1000 MHz (RIS Hi-Pave HR1 1000), and 2000 MHz (RIS Hi-Pave HR1 2000 and RIS Hi-Pave HR1 2000 NA) antenna frequencies, were used to collect data at $40 \mathrm{~cm}$ height from the surface of the ballast [48]. Concerning the $2000 \mathrm{MHz}$ antenna systems, one version for the European (EU) market (from now on, referred to as "2 GHz EU") and one low-powered antenna version for the North American (NA) market (from now on, referred to as "2 GHz NA") were used. Although the two mentioned antennas are centered around the same central frequency of investigation, the main difference lies in the radiated power of the antennas, which is lower in the case of the NA version. This is to comply with the regulatory policy [49] issued by the US Federal Communications Commission (FCC). In view of the lower radiative power, this antenna system exhibits worst performance in terms of SNR and, generally, the raw signal is characterized by relevant noise components that need to be filtered out. 512 sample size data scans with $15 \mathrm{~ns}\left(2.93 \times 10^{-2} \mathrm{~ns}\right)$ and $25 \mathrm{~ns}\left(4.89 \times 10^{-2} \mathrm{~ns}\right)$ time windows were collected using, respectively, the $1000 \mathrm{MHz}$ and the 2000 $\mathrm{MHz}$ systems. Limestone aggregate particles used for the construction of railway track beds were utilized for testing purposes. Geometric, mechanical and physical properties of the ballast aggregates were also assessed in the laboratory according to the EN 13450:2004 standard on railway ballast materials [50]. With regard to the pollutant material, a silty soil material classified as A4 by the AASHTO soil classification [51] was used and characterized according to relevant standard test methods. More information about the followed standard test methods and the material properties found for both the ballast and the pollutant materials can be found in Tosti et al. [19] and Benedetto et al. [28]. 
Figure 3 shows the combination of clean ballast (CB) and fouled ballast (FB) conditions used for reproducing the four scenarios in the laboratory environment. The samples have an effective height of $48 \mathrm{~cm}$. The Test Scenario "0" was representative of the clean ballast conditions (Figure 3(a)). 10 $\mathrm{cm}$ high layers consisting of a mix of pollutant A4 silty soil and ballast aggregates were manufactured and progressively increased from the bottom of the tank.
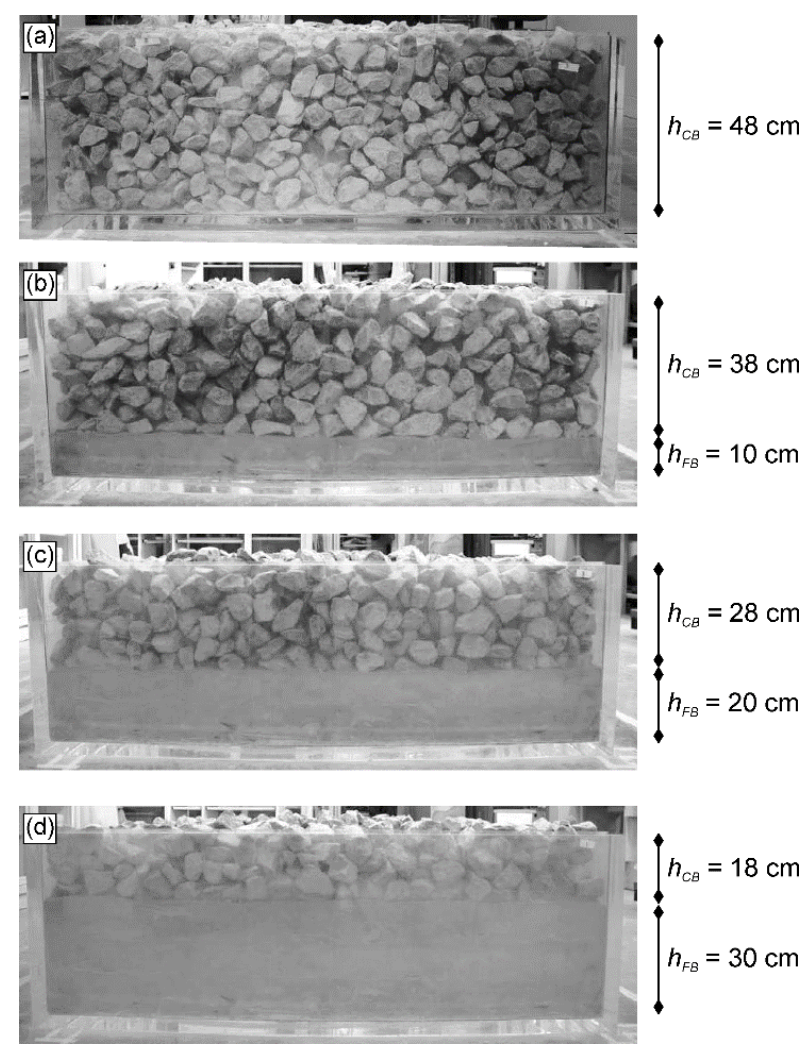

Fig. 3. Four scenarios of railway ballast/pollutant A4 soil manufactured in the laboratory, (a) Test Scenario "0": $48 \mathrm{~cm}$ clean ballast, (b) Test Scenario "I": $38 \mathrm{~cm}$ of clean ballast over $10 \mathrm{~cm}$ of fouled ballast, (c) Test Scenario "II":28 cm of clean ballast over $20 \mathrm{~cm}$ of fouled ballast, (d) Test Scenario "III": $18 \mathrm{~cm}$ of clean ballast over $30 \mathrm{~cm}$ of fouled ballast.

Thereby, different scenarios of fouling conditions were reproduced, namely, low (Figure 3(b) -Test Scenario "I": $h_{C B}=38 \mathrm{~cm} ; h_{F B}=10 \mathrm{~cm}$ ), medium (Figure 3(c) -Test Scenario "II": $h_{C B}=28 \mathrm{~cm} ; h_{F B}$ $=20 \mathrm{~cm}$ ) and high (Figure 3(d) -Test Scenario "III": $h_{C B}=18 \mathrm{~cm} ; h_{F B}=30 \mathrm{~cm}$ ). The laboratory procedure for the laying out of the material in the tank is described in Benedetto et al. [52].

\subsection{Experimental outcomes}

The objective of this Section is to confirm both the theoretical rationale and the numerical consistency of the proposed procedure by proving (as discussed below) a correlation between the 
entropy of a GPR datagram and the possible presence of fouling in the railway ballast. The reliability of the procedure is assessed in accordance with the aforementioned Neyman-Pearson test. The raw GPR data were used in order to lower the computational workload required for the implementation of the proposed entropy-based approach. Nevertheless, we have also verified with extensive laboratory experiments (here not reported for the sake of the compactness) that the advantage of filtering out mutual coupling effects from the raw GPR data is negligible. In the following results, the detection performance of the proposed approach has been assessed analytically, i.e. using (13) and (14) to theoretically evaluate the threshold and the corresponding probability of detection (according to the CFAR procedure), as well as by means of experimental based investigations in the laboratory. In particular, several empirical laboratory investigations have been carried out to estimate the actual threshold (determined by the desired probability of false alarm) and the probability of detection in order to validate the (asymptotic) results obtained by the theory. A commonly used method in the radar literature for the evaluation of the detection performance is based on the receiver's operating characteristic (ROC) curve. The ROC is graphically represented with diagrams, where $P_{F}$ is on the horizontal axis and $P_{D}$ is on the vertical one. Ideally, all the ROC must be above the $P_{D}=P_{F}$ line (bisector) and concave downward. Paradoxically, if they were not, a randomized test would be better. The best performing detector presents the minimum distance from the point $\left(P_{F}=0\right.$ and $\left.P_{D}=1\right)$ in its ROC curve. An effective operating point is just the point of the curve near such an optimum case.

Figure 4 reports the theoretically evaluated ROC of the ground-coupled radar system (equipped with $600 \mathrm{MHz}$ and $1600 \mathrm{MHz}$ monostatic antenna frequencies) for the three fouling scenarios of interest. The graph clearly shows that the $1600 \mathrm{MHz}$ antenna performs better than the $600 \mathrm{MHz}$ antenna in terms of detection performance, as it was theoretically expected. In particular, the performance of the $600 \mathrm{MHz}$ antenna is getting worse when passing from scenario I (where the clean ballast layer is thicker than the fouling ballast layer) to scenario III (where the clean ballast layer is thinner than the fouled ballast one). This is due to the resolution power of the $600 \mathrm{MHz}$ antenna (i.e. $12.5 \mathrm{~cm}$, as per the quarter of wavelength principle) and the configuration of scenario III where the ballast-pollutant mix is dominant with respect to the ballast-voids phase. 


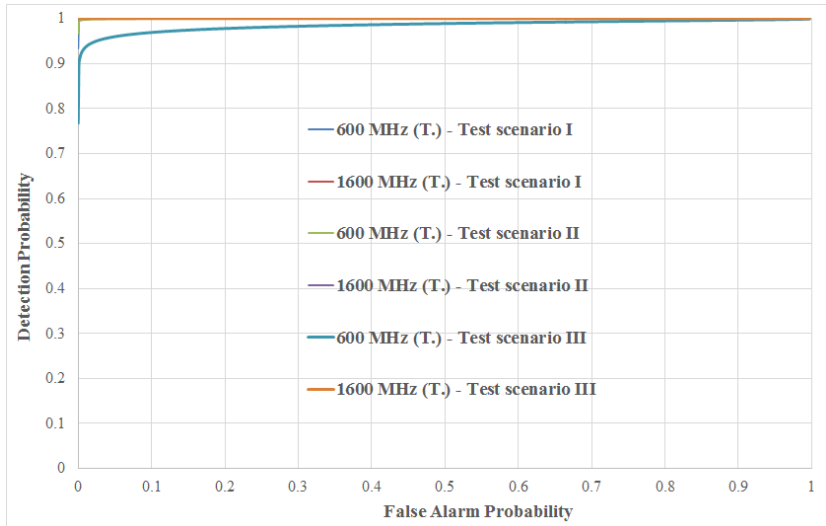

Fig. 4. Theoretical (T.) ROC of the ground-coupled GPR system, equipped with $600 \mathrm{MHz}$ and $1600 \mathrm{MHz}$ monostatic antenna frequencies, for the three scenarios of fouled ballast.

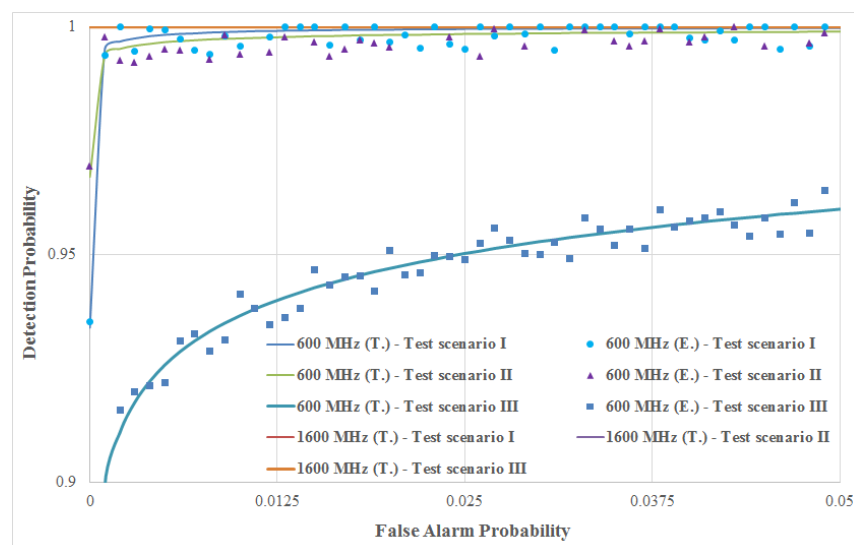

Fig. 5. Theoretical (T.) and Experimental (E.) ROC of the ground-coupled GPR system, equipped with $600 \mathrm{MHz}$ and $1600 \mathrm{MHz}$ monostatic antenna frequencies, for the three scenarios of fouled ballast of interest in the region $P_{D}=[0.9-1]$ and $P_{F}=[0-0.05]$.

Thereby, a more uniform character (i.e. fouled ballast) throughout the whole depth domain of the sample is observed at $600 \mathrm{MHz}$. Conversely, the quarter of wavelength resolution of the $1600 \mathrm{MHz}$ antenna (i.e. $4.7 \mathrm{~cm}$ ) still allows to detect scattering effects from the voids of the thinnest clean layer and to distinguish the fouled layer of the ballast (i.e., scenario III) [22]. Nevertheless, all the curves relatively overlap, and are characterized by detection performances greater than $95 \%$, with a target false alarm probability not exceeding the value of $2.5 \%$. Such a behaviour can be appreciated in Figure 5, where the region between $P_{D}=[0.9-1]$ and $P_{F}=[0-0.05]$ has been magnified. We have also added the empirically evaluated curves related to the $600 \mathrm{MHz}$ antenna, showing that the experimental results (obtained by the laboratory investigations) well match the theoretical results. Thereby, the validity of our proposed approach is confirmed. For the sake of the compactness, the experimentalbased curves for the $1600 \mathrm{MHz}$ antenna are here not showed since they completely overlap with the theoretical ones. 


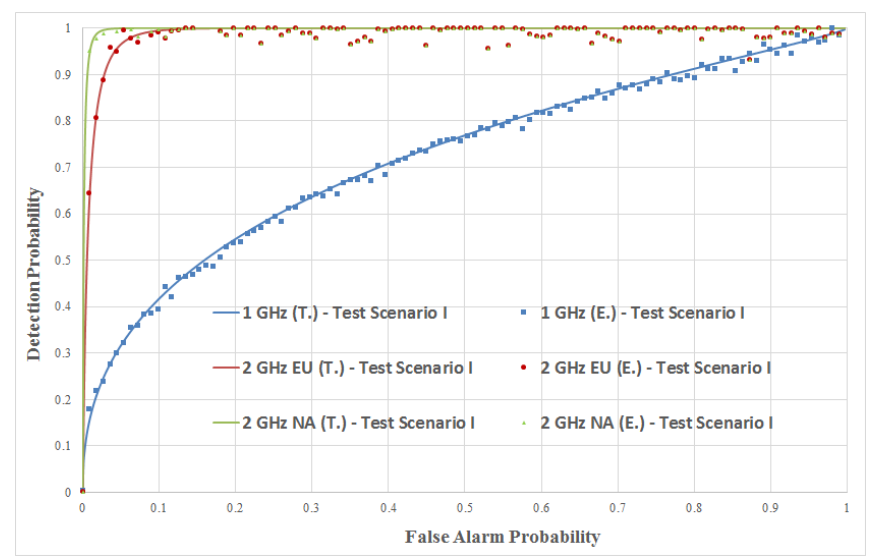

Fig. 6. Theoretical (T.) and Experimental (E.) ROC of the air-coupled GPR system, equipped with $1 \mathrm{GHz}, 2 \mathrm{GHZ} \mathrm{EU,}$ and $2 \mathrm{GHz}$ NA monostatic antenna frequencies, for the fouling scenario I.

Figures (6) - (8) illustrate the ROC of the air-coupled GPR equipped with $1 \mathrm{GHz}, 2 \mathrm{GHz} \mathrm{EU}$, and $2 \mathrm{GHz} \mathrm{NA}$ antenna frequencies for the three fouled scenarios, respectively. Again, the experimental (E.) results well match the theoretical (T.) curves; hence, the effectiveness of the mathematics in the previous sections are confirmed. Not surprisingly, in all the previous results different antennas provided different theoretical results. This is due to the fact that, as said before, the theoretical curves were obtained by means of eqs. (13)-(14). These two equations imply the presence of the two statistical moments (i.e. mean and variance) of the testing variable, that strictly depend on the characteristics of the signal under investigation (see [48] and references therein for further details). In all the analysed cases, the $1000 \mathrm{MHz}$ GPR system is characterized by the worst performance, again due to the lower resolution power of the selected antenna frequency $($ i.e. $7.5 \mathrm{~cm}$ ) as compared to the air-filled size of inter-granular voids. In particular, in the best case (i.e. scenario III in Figure (9)) the $1000 \mathrm{MHz}$ system reaches a detection probability higher than $90 \%$ only, with a corresponding false alarm rate equal to $50 \%$. Clearly, this is not a satisfactory operating condition. Conversely, both the $2000 \mathrm{MHz}$ systems (EU and NA antennas) perform better in the scenarios of interest. The $2 \mathrm{GHz} E U$ system has also proved to be relatively robust regarding the particular selected scenario. Indeed, considering the scenario I (Figure 6), II (Figure 7), and III (Figure 8), the $2 \mathrm{GHz}$ EU curve shows a detection probability always higher than $95 \%$, even with a relevant false alarm rate less than $5 \%$. Hence, this GPR system configuration and frequency of investigation are capable to identify ballast sections fouled with fine-graded material in 95 cases over 100, with an error rate less than 5 cases over 100. 


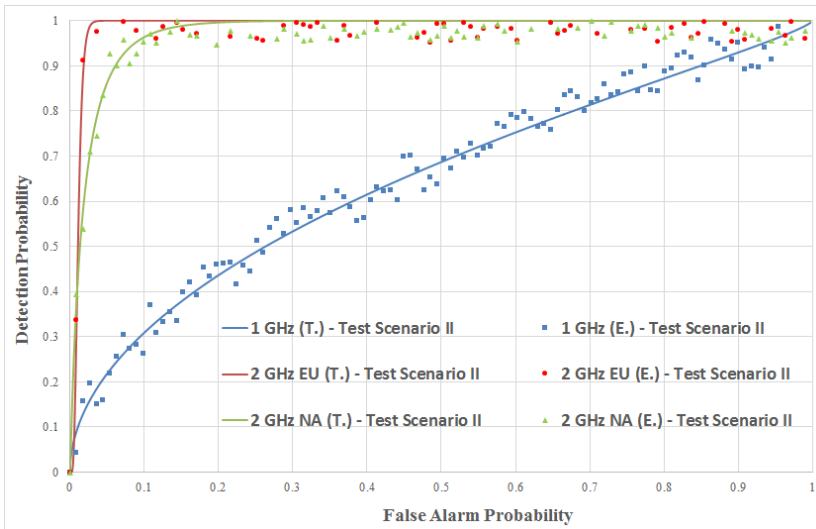

Fig. 7. Theoretical (T.) and Experimental (E.) ROC of the air-coupled GPR system, equipped with $1 \mathrm{GHz}, 2 \mathrm{GHZ}$ EU, and $2 \mathrm{GHz}$ NA monostatic antenna frequencies, for the fouling scenario II.

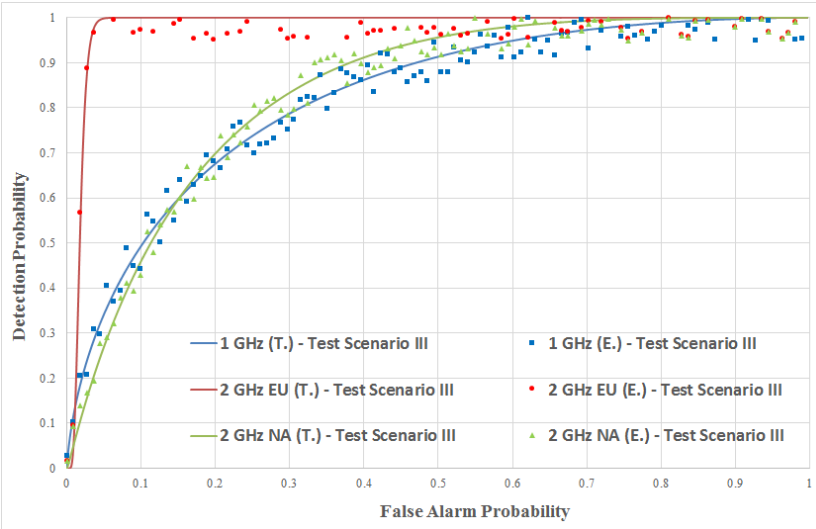

Fig. 8. Theoretical (T.) and Experimental (E.) ROC of the air-coupled GPR system, equipped with $1 \mathrm{GHz}, 2 \mathrm{GHZ} \mathrm{EU,}$ and $2 \mathrm{GHz}$ NA monostatic antenna frequencies, for the fouling scenario III.

This is a consequence of the fact that the $2000 \mathrm{MHz}$ GPR system has a resolution power of $3.75 \mathrm{~cm}$ (quarter of wavelength criterion), which is most suited to detect scattering effects from voids [22]. Finally, it is important to emphasize how the $2 \mathrm{GHz} N A$ antenna has similar performance in the first two scenarios, whereas it greatly lowers its detection capability in the case of the thickest fouled layer (i.e. scenario III). This may be likely due to the different characteristics of this system, being the 2 GHz NA a lower powered antenna, whereby clutter is more sensitive to the effects caused by the returns from the target [37].

\section{Conclusion And Future Perspectives}

This paper reports on a signal processing procedure based on an entropy analysis which has been innovatively applied to ground penetrating radar (GPR) data for the assessment and the health 
monitoring of railway ballast conditions. In order to simulate a real-life scenario within the context of railway structures, four different ballast/pollutant mixes were introduced from clean to highly-fouled ballast conditions. Limestone aggregate particles used for the construction of railway track beds and a silty soil material classified as A4 by the AASHTO soil classification [51] were used for testing purposes. The experimental tests were carried out using GPR systems equipped with different groundcoupled (600 and $1600 \mathrm{MHz}$ ) and air-coupled antennas (1000 and $2000 \mathrm{MHz}$ ). With regard to the $2000 \mathrm{MHz}$ radar systems, one ordinary (i.e., $2 \mathrm{GHz} \mathrm{EU}$ ) and one low-powered (i.e., $2 \mathrm{GHz} \mathrm{NA}$ ) version of the horn antenna for the European (EU) and the North-American (NA) markets were used, respectively.

A binary hypothesis testing method that automatically discriminates between the presence and the absence of fouled ballast was proposed. The performance of this method was evaluated in terms of the receiver's operating characteristic (ROC) (i.e. the detection probability versus the false alarm rate) by the calibration of an optimal threshold set from the outcomes of laboratory tests. The agreement between the theoretically-based and the experimental-based results confirms the validity of the proposed approach at rapidly identifying distinctive areas of interest related to fouling. Thereby, the computational costs traditionally related to more sophisticated data processing could be considerably lowered. On a comparable (multi-frequency) GPR system with ground-coupled antennas, the lowfrequency antenna (i.e. $600 \mathrm{MHz}$ ) turned out to provide a lower detection performance than the higher frequency (i.e. $1600 \mathrm{MHz}$ ). Such an outcome was confirmed by the application of the proposed entropy-based approach to the data collected using the air-coupled GPR systems. Both the $2000 \mathrm{MHz}$ systems have returned detection probability values higher than the $1000 \mathrm{MHz}$. In particular, the $2 \mathrm{GHz}$ EU version of the antenna has proved to be the most effective within the context of the entropy-based analyses here proposed, showing a detection probability always higher than $95 \%$ and a relevant false alarm rate less than 5\%. The results proved how the use of frequencies of investigation greater than $1600 \mathrm{MHz}$ is more suitable for the inspection of ballast by pursuing the proposed entropy-based methodology. Resolution powers with dimensions consistent with the size of the inter-particle voids allow to detect the thinner clean layers of the top ballast as well as to distinguish these from the underlying fouled layers.

To this effect, the study confirms the effectiveness of the method in the assessment of the railway ballast conditions and it suggests effective frequencies of investigation to be purposely selected. By means of the proposed methodology, distinctive areas of interest related to the fouling intrusion could 
be rapidly identified, thereby lowering significantly the amount of data to be processed as well as the time required for specialist data processing.

It is worth mentioning that the proposed methodology is planned to be tested on real-life railway track beds in the future. To this effect, the reflection recorded from the metallic reflector will have to be very likely represented by the dielectric contrast between the ballast/sub-ballast layer and the foundation (subgrade) soil.

\section{ACKNOWLEDGEMENT}

The authors are grateful to Mr. Spartaco Cera, from Roma Tre University, for the technical assistance during the laboratory tests and for providing invaluable help in this study. Special thanks to IDS Georadar for supplying part of the radar systems, and Clax Italia s.r.l. for manufacturing the methacrylate tank. This work has also benefited from the network activities carried out within the EUfunded COST Action TU1208 “Civil Engineering Applications of Ground Penetrating Radar”.

\section{REFERENCES}

[1] Indraratna, B. 1st Ralph Proctor Lecture of ISSMGE. Railroad performance with special reference to ballast and substructure characteristics. Transportation Geotechnics, 7, pp. 74-114, 2016.

[2] Selig, E. T. \& Waters, J. M. Track geotechnology and substructure management. Thomas Telford, London, 1994

[3] Anbazhagan, P., Lijun, S., Buddhima, I., Cholachat, R. Model track studies on fouled ballast using ground penetrating radar and multichannel analysis of surface wave. Journal of Applied Geophysics, 74 (4), pp. 175-184, 2011.

[4] I. L. Al-Qadi, S Zhao, P. Shangguan. Railway Ballast Fouling Detection Using GPR Data: Introducing a Combined TimeFrequency and Discrete Wavelet Techniques. Near Surface Goephysics, vol. 14, no. 2, pp. 145-153, 2016.

[5] Anbazhagan, P., Dixit, P.S.N., Bharatha, T.P. Identification of type and degree of railway ballast fouling using ground coupled GPR antennas, Journal of Applied Geophysics, vol. 126, pp. 183-190, 2016.

[6] De Bold, R., O'Connor, G., Morrissey, J.P., Forde, M.C. Benchmarking large scale GPR experiments on railway ballast, Construction and Building Materials, 92, art. no. 5926, pp. 31-42, 2015.

[7] Feldman, F., and Nissen, D. Alternative testing method for the measurement of ballast fouling: percentage void contamination. In Proceedings of the Conference on Railway Engineering. Railway Technical Society of Australia, Canberra, Australia. pp. 101-109, 2002.

[8] Indraratna, B., Su, L., Rujikiatkamjorn, C. A new parameter for classification and evaluation of railway ballast fouling, Technical note. Can. Geotech. J. 48, 322-326, 2011.

[9] Clark, M., McCann, D.M., Forde, M.C. Infrared thermographic investigation of railway track ballast. NDT and E International, 35 (2), pp. 83-94, 2002. 
[10] Anbazhagan, P., Buddhima, I., Amarajeevi, G. Characterization of clean and fouled rail track ballast subsurface using seismic surface survey method: Model and field studies. Journal of Testing and Evaluation, 39 (5), pp. 1-11, 2011.

[11] Donohue, S., Gavin, K., Tolooiyan, A. Geophysical and geotechnical assessment of a railway embankment failure. Near Surface Geophysics, 9 (1), pp. 33-44, 2011.

[12] Hugenschmidt J. Railway track inspection using GPR. Journal of Applied Geophysics, 43: 147-155, 2000.

[13] Clark, M.R, Gillespie, R., Kemp, T., McCann, D.M, Forde, M.C. Electromagnetic properties of railway ballast. NDT and E International, 34 (5), pp. 305-311, 2001.

[14] Al-Qadi, I.L., Xie, W., Roberts, R. Scattering analysis of ground-penetrating radar data to quantify railroad ballast contamination. NDT and E International, 41 (6), pp. 441-447, 2008.

[15] R. Roberts, A. Schutz, I.L. Al-Qadi, E. Tutumluer, Characterizing railroad ballast using GPR: recent experiences in the United States. In Proc. of the 2007 4th International Workshop on Advanced Ground Penetrating Radar (IWAGPR 2007), Naples, Italy, Jun. 2007.

[16] Railway Track and Structures Magazine, June 1985.

[17] Daniels D.J., Ground Penetrating Radar, 2nd Edition. London, U.K.: The Inst. Electrical Eng., 2004.

[18] Sussmann T. R., O’Hara K.R., and Selig E.T. Development of material properties for railway application of ground penetrating radar. In Proc. of the Society of Photo-Optical Instrumentation Engineers (SPIE), vol. 4758, 2002.

[19] Tosti F., Benedetto A., Calvi A., and Bianchini Ciampoli L. Laboratory Investigations for the Electromagnetic Characterization of Railway Ballast through GPR. In Proc. 16th International Conference of Ground Penetrating Radar (GPR 2016), Hong Kong, June 13-16, 2016.

[20] Fontul, S., Fortunato, E., De Chiara, F. Evaluation of ballast fouling using GPR. In Proceedings of the 15th International Conference on Ground Penetrating Radar, GPR 2014, art. no. 6970458, pp. 418-422, 2014

[21] P. Anbazhagan, P.S. Naresh Dixit, T.P. Bharatha, Identification of type and degree of railway ballast fouling using ground coupled GPR antennas. Journal of Applied Geophysics, vol. 126, pp. 183-190, 2016.

[22] R. Roberts, J. Rudy, I. Al-Qadi, E. Tutumluer. Railroad Ballast Fouling Detection Using Ground Penetrating Radar - A New Approach Based on Scattering from Voids. Ninth European Conference on NDT, ECNDT 2006 - Th.4.5.1, 2006.

[23] M.C. Forde, R. De Bold, G. O’Connor, J.P. Morrissey. New analysis of Ground Penetrating Radar testing of a mixed railway trackbed. Transportation Research Board Annual Meeting, 2010.

[24] Leng, Z., Al-Qadi, I.L. Railroad ballast evaluation using ground-penetrating radar . Transportation Research Record, (2159), pp. 110-117, 2010.

[25] W. Shao, A. Bouzerdoum, S.L. Phung, L. Su, B. Indraratna, C. Rujikiatkamjorn. Automatic classification of groundpenetrating-radar signals for railway-ballast assessment. Geoscience and Remote Sensing IEEE Transactions on, 49 (10), pp. 3961-3972, 2011.

[26] J. Xiao and L. Liu. Multi-frequency GPR signal fusion using forward and inverse S-transform for detecting railway subgrade defects. In Proc. of 8th International Workshop on Advanced Ground Penetrating Radar (IWAGPR), Florence, pp. 1-4, 2015.

[27] Q. Zhang, J. Gascoyne and A. Eriksen. Characterisation of ballast materials in trackbed using ground penetrating Radar: Part 1. In Proc. of 5th IET Conference on Railway Condition Monitoring and Non-Destructive Testing (RCM 2011), pp. 1-8, 2011. 
[28] A. Benedetto, F. Tosti, L. Bianchini Ciampoli, L. Pajewski, D. Pirrone, A. Umiliaco, M.G. Brancadoro. A simulation-based approach for railway applications using GPR. In Proc of the 2016 International Conference of Ground Penetrating Radar, Hong Kong, 13-16 June, 2016.

[29] A. Benedetto, F. Benedetto, M. R. De Blasiis, G. Giunta. Reliability of signal processing technique for pavement damages detection and classification using ground penetrating radar. IEEE Sensors Journal, vol. 5, no. 3, pp. 471-480, 2005.

[30] T.K. Sarkar, H. Schwarzlander, Seungwon Choi, M.S. Palma, M.C. Wicks, "Stochastic versus deterministic models in the analysis of communication systems", IEEE Antennas and Propagation Mag., vol. 44, no. 4, pp. 40-50, 2002.

[31] Z. Thian, E. Blasch, "Compressed sensing for MIMO radar: A stochastic perspective", IEEE Statistical Signal Processing Workshop (SSP), 2012.

[32] R.M. Howard, A Signal Theoretic Introduction to Random Processes. Wiley, 2016.

[33] S. Miller, D. Childers. Probability and Random Processes: With Applications to Signal Processing and Random Processes. Academic Press, 2012.

[34] A.V. Oppenheim, R.W. Schafer, Digital Signal Processing. Prentice-Hall, Upper Saddle River, NJ (1975).

[35] A. Benedetto. Water content evaluation in unsaturated soil using GPR signal analysis in the frequency domain. J. Appl. Geophys., vol. 71, no.1, pp. 26-35, 2010.

[36] A. Benedetto, F. Benedetto, and F. Tosti. GPR applications for geotechnical stability of transportation infrastructures. Nondestruct. Test. Eva., vol. 27, no. 3, pp. 253-262, 2012.

[37] F. Benedetto and F. Tosti. GPR spectral analysis for clay content evaluationvby the frequency shift method. J. Appl. Geophys., vol. 97, pp. 89-96, 2013.

[38] F. Tosti, A. Benedetto, L. B. Ciampoli, S. Lambot, C. Patriarca, and E. C. Slob. GPR analysis of clayey soil behaviour in unsaturated conditions for pavement engineering and geoscience applications. Near Surf. Geophys., vol. 14, no. 2, pp. $127-$ $144,2016$.

[39] Jaynes, E.T. Gibbs vs Boltzmann entropies. Am. J. Phys. 33 (5), 391-398, 1965.

[40] C.E. Shannon. A mathematical theory of communication. Bell Syst. Tech. J., 27, pp. 379-423 623-656, 1948.

[41] Zhang, Y., Candra, P., Wang, G., Xia, T. 2-D fourier transform to leverage GPR data analysis efficiency. IEEE Transactions on Instrumentation and Measurement, 64 (1), art. no. 6850057, pp. 103-111, 2015.

[42] J García-Palacios, A Samartín, M Melis. Analysis of the railway track as a spatially periodic structure. Proceedings of the Institution of Mechanical Engineers, Part F: Journal of Rail and Rapid Transit, vol. 226, no. 2, 2012.

[43] J.P. Burg, Maximum entropy spectral analysis, Proc. 37th Ann. Meet. Soc. Explor. Geophys. 1967.

[44] J.P. Burg, Maximum entropy analysis, Ph.D. dissertation Stanford Univ., Stanford, CA, 1975.

[45] B.S. Choi, T.M. Cover, An information-theoretic proof of Burg's maximum entropy spectrum, Proc. IEEE, 72 (8), pp. 10941096, 1984.

[46] Y. Inouye, Maximum entropy spectral estimation for regular time series of degenerate rank. IEEE Trans. Acoust. Speech Signal Process., 32 (4), pp. 733-740, 1984.

[47] H. L. Van Trees, Detection, Estimation, and Modulation Theory. New York: Wiley, 1971.

[48] F. Benedetto, F. Tosti, A signal processing methodology for assessing the performance of ASTM standard test methods for GPR systems. Signal Processing (Elsevier), vol. 132, pp. 327-337, 2017. 
[49] Federal Communications Commission Office of Engineering \& Technology. 1997. Evaluating Compliance with FCC Guidelines for Human Exposure to Radiofrequency Elec-tromagnetic Fields, OET Bulletin 65, Edition 97-01.

[50] EN 13450:2002/AC:2004. Aggregates for railway ballast. European Committee for Standardization, 2004.

[51] American Association of State Highway and Transportation Officials (AASHTO) 2011. Roadside Design Guide, 4th edn. AASHTO, Washington, DC.

[52] A. Benedetto, F. Tosti, L. Bianchini Ciampoli, A. Calvi, M.G. Brancadoro, A.M. Alani. Electromagnetic characterization of clean and fouled railway ballast through GPR signal processing and numerical simulation. Construction and Building Materials, Submitted, 2016. 